\title{
A QUASI-STATIONARY SOLUTION TO GLIESE 436b’s ECCENTRICITY
}

\author{
Konstantin Batygin $^{1,2}$, Gregory Laughlin ${ }^{1}$, Stefano Meschiari $^{1}$, Eugenio Rivera $^{1}$, Steve $_{\text {Vogt }}{ }^{1}$, and Paul Butler $^{3}$ \\ ${ }^{1}$ UCO/Lick Observatory, University of California, Santa Cruz, CA 95064, USA \\ ${ }^{2}$ Division of Geological and Planetary Sciences, California Institute of Technology, Pasadena, CA 91125, USA \\ ${ }^{3}$ Department of Terrestrial Magnetism, Carnegie Institution of Washington, Washington, DC 20015, USA \\ Received 2009 January 13; accepted 2009 April 20; published 2009 June 9
}

\begin{abstract}
We investigate the possibility that the large orbital eccentricity of the transiting Neptune-mass planet Gliese $436 \mathrm{~b}(\mathrm{Gl} \mathrm{436b})$ is maintained in the face of tidal dissipation by a second planet in the system. We find that the currently observed configuration can be understood if $\mathrm{Gl} 436 \mathrm{~b}$ and a putative companion have evolved to a quasistationary fixed point in which the planets' orbital apses are co-linear and in which secular variations in the orbital eccentricities of the two planets have been almost entirely damped out. In our picture, the two planets are currently experiencing a long period of gradual orbital circularization. Specifically, if Gl $436 \mathrm{~b}$ has a tidal $Q \sim 300,000$, similar to both the Jovian $Q$ and to the upper limit for the Neptunian $Q$, then this circularization timescale can be of order $\tau \sim 8$ Gyr given the presence of a favorably situated perturber. We adopt an octopole-order secular theory based on a Legendre expansion in the semimajor axis ratio $a_{1} / a_{2}$ to delineate well-defined regions of $\left(P_{c}, M_{c}, e_{c}\right)$ space that can be occupied by a perturbing companion. This description includes the leading-order effects of general relativity, and retains accuracy for perturbing companion planets that have high eccentricity. We incorporate the evolutionary effect of tidal dissipation into our secular model of the system, and solve the resulting initial value problems for a large sample of the allowed configurations. We find a locus of apsidally aligned configurations that are (1) consistent with the currently published radial velocity data, (2) consistent with the current lack of observed transit timing variations (TTVs), (3) subject to rough constraint on dynamical stability, and which (4) have damping timescales consistent with the current multi-Gyr age of the star. We then polish the stationary configurations derived from secular theory with full numerical integrations, and compute the TTVs and radial velocity half-amplitudes induced by the resulting configurations. We present our results in the form of candidate companion planets to Gl 436b. For these candidates, radial velocity half-amplitudes, $K_{c}$, are of order $3 \mathrm{~m} \mathrm{~s}^{-1}$, and the maximum amplitude of orbit-to-orbit TTVs are of order $\Delta t=1 \mathrm{~s}$ to $\Delta t=5 \mathrm{~s}$. For the particular example case of a perturber with orbital period, $P_{c}=40 \mathrm{~d}$, mass, $M_{c}=8.5 M_{\oplus}$, and eccentricity, $e_{c}=0.58$, we confirm our semianalytic calculations with a full numerical three-body integration of the orbital decay that includes tidal damping and spin evolution. Additionally, we discuss the possibility of many-perturber stationary configurations, utilizing modified Laplace-Lagrange secular theory. We then perform a proof-of-concept tidally dissipated numerical integration with three planets, which shows the system approaching a triply circular state.
\end{abstract}

Key words: methods: analytical - methods: numerical - planetary systems

\section{INTRODUCTION}

In the years since the discovery of the planetary companion to 51 Peg (Mayor \& Queloz 1995), considerably fewer than 1000 additional extrasolar planets have been detected. ${ }^{4}$ Because the aggregate of known planets is still quite limited, the discovery and characterization of additional planets orbiting specific individual stars, remains a topic of considerable interest.

The detection of Gliese 436b (Gl 436b) by Butler et al. (2004) marked the first discovery of a Neptune-mass planet since LeVerrier's discovery of Neptune itself in 1846. In 2007 May, Gl 436b was discovered to be observable in transit (Gillon et al. 2007) which has sparked an intense interest in this remarkable planet. The transits resolve the $\sin (i)$ degeneracy, and fix the mass of the planet at $m_{b}=23.17 \pm 0.79 m_{\oplus}$ (Torres 2007). The period of the planet is $P=2.643904 \pm 0.000005 \mathrm{~d}$ (Bean et al. 2008) and it orbits an $M_{\star}=0.452_{-0.012}^{+0.014} M_{\odot}$ red dwarf primary (Torres 2007). To date, no timing variations have been observed for the transits of Gl 436b, with strict orbital periodicity maintained to a level of at least $\sim 7 \mathrm{~s}$ (Pont et al. 2009).

The radius, $R_{b}=4.22_{-0.10}^{+0.09} R_{\oplus}$ (Torres 2007) of Gl 436b indicates that it necessarily contains several Earth masses of

\footnotetext{
4 For an up-to-date listing of the current galactic planetary census, see http://www.exoplanet.eu
}

hydrogen and helium in its outer layers. It probably resembles Neptune in overall composition (see, e.g., the models of Fortney et al. 2007). Measurement of the secondary eclipse depth by the Spitzer Space Telescope (Deming et al. 2007; Demory et al. 2007) indicates that the planet has an $8 \mu \mathrm{m}$ brightness temperature of $T=712 \pm 36 \mathrm{~K}$. If we adopt $T_{\text {eff }}=3350 \mathrm{~K}$ for Gliese 436A, take a zero albedo for the planet, and assume a uniform reradiation of the orbit-averaged received flux from the entire planetary surface, we obtain a planetary effective temperature $T_{\mathrm{eq}}=642 \mathrm{~K}$. The somewhat higher temperature implied by the secondary eclipse depth could arise from inefficient transport of heat to the night side of the planet, from a non-blackbody planetary emission spectrum, from tidal luminosity, or from a combination of the three. The possibility that tidal luminosity is being observed is prompted by the orbital phase, $\phi=0.587$, of the secondary eclipse, which confirms that the orbital eccentricity is alarmingly high (with a best-fit value of $e=0.150 \pm 0.012$; Deming et al. 2007). Indeed, the planet and its primary bring to mind an oversize alien version of Jupiter and Io.

The short orbital period and large eccentricity of Gl 436b suggest that tidal circularization might be highly effective. To second order in eccentricity, the tidal luminosity of a spinsynchronous planet (Peale \& Cassen 1978; Mardling 2007) is 
given by

$$
\frac{d E}{d t}=\frac{21}{2} \frac{k_{2}}{Q} \frac{G M_{\star}^{2} n R_{b}^{5} e^{2}}{a^{6}},
$$

where $k_{2}$ is the planetary tidal Love number, $a$ is the semimajor axis, $n$ is the orbital mean motion, and $Q$ is the planet's effective tidal dissipation parameter. Note that Gl 436b cannot be fully spin synchronized, and because it presumably has no permanent quadropole moment, it should not reside in spin-orbit resonance. The pseudosynchronization theory of Hut (1981; see also Goldreich \& Peale 1966) suggests $P_{\text {spin }}=2.32$ d, leading to a 19-d synodic period for the planet $b$. The analysis of Levrard et al. (2007), furthermore, indicates that this spin asynchronicity of the planet will cause the tidal luminosity to exceed that given by the above formula by a small amount.

To date, several authors have investigated the Gliese 436 system with an eye toward obtaining a convincing explanation for planet b's large eccentricity. To establish context, it is useful to review these contributions.

\subsection{Solution 1: A Large $Q$}

Jackson et al. (2008b) have performed tidally dissipated single-planet integrations backward in time, and have shown that $Q_{b} / k_{2 b} \approx 10^{6.6}$ and $Q_{\star} / k_{2 \star} \approx 10^{5.5}$ can explain the high eccentricity of $\mathrm{Gl} 436 \mathrm{~b}$. However, are such $Q$ values realistic? The resonant configuration of the Jovian satellites implies $10^{5}<Q_{\mathrm{J}}<2 \times 10^{6}$, with the preferred value closer to the lower limit (Goldreich \& Soter 1966; Peale \& Greenberg 1980). Meyer \& Wisdom (2008) argue that $Q_{\mathrm{S}}<10^{5}$ whereas the circularization boundary for extrasolar planets (Wu 2003) suggests typical $Q$ values of order $3 \times 10^{5}$, allowing for significant variation from planet to planet in addition to significant overall uncertainty. Uranus and Neptune, which more closely resemble $\mathrm{Gl} 436 \mathrm{~b}$ in mass, composition, and radius, are a further factor of approximately 10 times more dissipative. Tittemore \& Wisdom (1989) have demonstrated that $Q_{\mathrm{U}} \lesssim 3.9 \times 10^{4}$, whereas in the case of Neptune, Banfield \& Murray (1992) find $1.2 \times 10^{4}<Q_{\mathrm{N}}<3.3 \times 10^{5}$. Recent calculations of Zhang \& Hamilton (2008) suggest an even lower estimate: $9 \times 10^{3}<Q_{\mathrm{N}}<3.6 \times 10^{4}$.

A theory for the origin of planetary $Q$ values (not to speak of a definitive understanding of dynamical tides) remains elusive, and so there is no a priori reason to reject the hypothesis that G1 $436 \mathrm{~b}$ has a remarkably small internal dissipation. It should be noted, however, that for the particular case of Gl 436b, $Q$ would need to be even higher than the Jackson et al. (2008b) estimate of $Q_{b} / k_{2 b} \approx 10^{6.6}$, given the likely breakdown of second-order equilibrium tidal theory at the very high initial eccentricities that they considered (Wisdom 2008).

\subsection{Solution 2: A Resonant Perturber}

A natural solution to the high-eccentricity problem is to invoke perturbations from an as-yet undetected second planet in the system. Ribas et al. (2008) have suggested that the perturbing body lies in a 2:1 mean motion resonance (MMR) with the primary planet. By constructing a two-planet fit to the radial velocity data of Maness et al. (2007), they produced a specific orbital model for the second planet, and predicted that changes in the eclipse depth and duration (arising from precession of Gl $436 \mathrm{~b}$ driven by the interaction with c) should be observable.

Resonant perturbations contribute to the disturbing function with terms that contain mean longitudes, $\lambda$, and so they are generally capable of producing far larger transit timing variations
(TTVs) than secular perturbations. Indeed, most discussions of the transit timing technique have focused on perturbing planets in low-order mean motion resonances (e.g., Agol et al. 2005; Holman \& Murray 2005). In the context of Gliese 436, the Ribas et al. (2008) model generates TTVs measured in minutes, making their hypothesis eminently testable.

The $2.64 \mathrm{~d}$ period of planet $\mathrm{b}$ allows transit measurements to accumulate rapidly, and during the time that has elapsed since the Gillon et al. (2007) discovery, a number of high-precision observations of transit midpoint times have become publicly available. Alonso et al. (2008) analyzed the archival Spitzer photometry obtained via "target of opportunity" observations in 2007 June, and derived $T_{c}=2454280.78191 \pm 0.00028 \mathrm{HJD}$ (24 s accuracy). The same authors also analyzed their own ground-based observations of the 2008 March 8 transit, and derived $T_{c}=2454534.59584 \pm 0.00015 \mathrm{HJD}$ (13 s accuracy). By comparing these transit times with the Gillon et al. (2007) discovery ephermeris of $T_{c}=2454222.616 \pm 0.001 \mathrm{HJD}(86 \mathrm{~s}$ accuracy), Alonso et al. (2008) then determined that there is only a $\sim 0.5 \%$ probability that a $5 M_{\oplus}$ perturber in an exterior 2:1 mean motion resonance would have produced transit TTVs smaller than those implied by the three midpoint times in their analysis. Recent work by Pont et al. (2009) has pushed the threshold for TTVs below $7 \mathrm{~s}$, ruling out perturbers in 2:1 resonance down to lunar mass. Mardling (2008), furthermore, shows that semimajor axis decay produced by tidal dissipation will cause any 2:1 resonance between GL436b and a hypothetical companion to be short lived. In particular, a perturber with the properties proposed by Ribas et al. (2008) passes through a 2:1 resonance on a timescale of $\tau_{p}=0.3\left(Q_{b} / 10^{5}\right) \mathrm{Gyr}$.

\subsection{Solution 3: A Secular Perturber}

In a recent paper, Tong \& Zhou (2009) explored Gl 436b's eccentricity modulation by both secular and resonant companions. Their approach adopted an octopole-order secular theory (see below) to identify hypothetical perturbers that can excite planet b's eccentricity (in the course of a single secular cycle) from $e_{b}=0$ to $e_{b}=0.16$, while simultaneously requiring that the system as a whole remain dynamically stable and that the perturber induces a radial velocity half-amplitude less than the current threshold of detection (which they took to be $K<3 \mathrm{~m} \mathrm{~s}^{-1}$ ). Interestingly, they located a range of candidate perturbers that satisfied the constraints. Tong \& Zhou (2009) found, however, that when the effects of tidal dissipation were incorporated and the hypothetical two-planet systems were integrated forward in time, all eccentricity modulation was damped out and the orbits quickly circularized. As a part of their survey, Tong \& Zhou (2009) also specifically exclude the class of perturbing companion planets suggested by Maness et al. (2007), noting, for example, that a putative perturber with $M_{c}=0.12 M_{\text {Jup }}$, $P=14 \mathrm{yr}$, and $e_{c}=0.6$ is capable of exciting an eccentricity, $e_{b_{\max }}$, for Gl $436 \mathrm{~b}$ to only $3 \times 10^{-6}$.

The aggressively effective orbital circularization observed by Tong \& Zhou (2009) led those authors to conclude that a companion planet (exerting either resonant or secular perturbations) that is consistent with the current corpus of Doppler velocity observations "cannot excite and maintain the significant eccentricity" of G1 436b. They argue that in order to adequately explain Gl 436b's non-circular orbit, its tidal quality factor must be of order $Q_{b} / k_{2 b} \sim 6 \times 10^{6}$, a factor likely an order of magnitude larger than the values generally ascribed to gas giants (see, however, Jackson et al. 2008a who argue that the upper limits on 
Jupiter's $Q$ may be significantly higher than generally believed, and see Matsumura et al. 2008 who present evidence of high $Q$ for the aggregate of known extrasolar planets).

In any case, the non-circular orbit of Gl $436 \mathrm{~b}$ constitutes a remarkable puzzle. The solution likely has interesting implications for our understanding of planetary structure, formation, and dynamics. The problem is rendered even more worthwhile by the fact that improved spectroscopic and photometric observations can be used to test the predictions inherent in a particular solution, as has been demonstrated already with the Ribas et al. (2008) hypothesis. This paper reports our attempt at a dynamical solution using currently published observational constraints. We investigate an alternate hypothesis that while the perturbations are secular in nature, the eccentricity is not modulated, and the orbits of both planets are quasi-stationary. We present a semianalytical survey which demonstrates that a range of welldefined apsidally aligned perturbers are individually capable of prolonging the effective circularization timescale of Gl $436 \mathrm{~b}$ by more than a factor of 5 . We use numerical integrations to correct for planet-planet dynamics not captured by our secular theory, and present our final locus of predictions for the perturber properties in the form of a table. We confirm the long-term evolution for a select test case with a fully numerical scheme, and we discuss the possibilities for near-term observational confirmation. Finally, we discuss the possibility of a multiple-perturber scenario in the context of a modified Laplace-Lagrange secular theory and perform one proof-of-concept numerical integration.

\section{THE DYNAMICAL STATE OF THE SYSTEM}

We first investigate the current dynamical state of a twoplanet system. Throughout out analysis, we assume that the inclinations of perturbing planets with respect to Gl 436b's orbit are dynamically negligible. This assumption may turn out to be overly restrictive, but we note that if the orbital angular momentum vector of $\mathrm{Gl} 436 \mathrm{~b}$ is found to be in near alignment with the rotation axis of the parent star, then the possibility that the system is nearly co-planar is strengthened. To date, however, there have been no reported observations of the Rossiter-McLaughlin effect (see, e.g., Winn et al. 2005) during transits of Gl 436b.

In the absence of mean motion resonances, the dynamics of a stable multiplanet system are dominated by secular interactions, with tidal forces also playing a significant role. In this scenario, any initial secular variations of the planets' orbital elements are damped out over $\sim 3$ tidal circularization timescales for the inner planet, given by

$$
\tau_{b}=\left(\frac{G M_{\star} m_{b}}{a_{b}}\right)\left(\frac{e_{b}^{2}}{\dot{E}}\right),
$$

and the system approaches a quasi-stationary state. Once the fixed point is reached, the system begins to evolve exponentially toward a doubly circular state over a timescale that is considerably longer than the circularization timescale (Mardling 2007).

Correcting for the leading-order effects of general relativity (GR), which are crucial to this system, the orbit-averaged rates of change of eccentricities and longitudes of perihelia of the coplanar system are given by (Mardling \& Lin 2002; Mardling 2007)

$$
\frac{d e_{b}}{d t}=-\frac{15}{16} n_{b} e_{c}\left(\frac{m_{c}}{M_{\star}}\right)\left(\frac{a_{b}}{a_{c}}\right)^{4} \frac{\sin \left(\varpi_{b}-\varpi_{c}\right)}{\left(1-e_{c}^{2}\right)^{5 / 2}},
$$

$$
\begin{gathered}
\frac{d e_{c}}{d t}=\frac{15}{16} n_{c} e_{b}\left(\frac{m_{b}}{M_{\star}}\right)\left(\frac{a_{b}}{a_{c}}\right)^{3} \frac{\sin \left(\varpi_{b}-\varpi_{c}\right)}{\left(1-e_{c}^{2}\right)^{2}} \\
\frac{d \varpi_{b}}{d t}=\frac{3}{4} n_{b}\left(\frac{m_{c}}{M_{\star}}\right)\left(\frac{a_{b}}{a_{c}}\right)^{3} \frac{1}{\left(1-e_{c}^{2}\right)^{3 / 2}}\left[1-\frac{5}{4}\left(\frac{a_{b}}{a_{c}}\right)\left(\frac{e_{c}}{e_{b}}\right)\right. \\
\left.\times \frac{\cos \left(\varpi_{b}-\varpi_{c}\right)}{1-e_{c}^{2}}\right]+\frac{3 n_{b}^{3}}{1-e_{b}^{2}}\left(\frac{a_{b}}{c}\right)^{2}
\end{gathered}
$$

and

$$
\begin{aligned}
\frac{d \varpi_{c}}{d t}= & \frac{3}{4} n_{c}\left(\frac{m_{b}}{M_{\star}}\right)\left(\frac{a_{b}}{a_{c}}\right)^{2} \frac{1}{\left(1-e_{c}^{2}\right)^{2}}\left[1-\frac{5}{4}\left(\frac{a_{b}}{a_{c}}\right)\left(\frac{e_{b}}{e_{c}}\right)\right. \\
& \left.\times \frac{\left(1+4 e_{c}^{2}\right)}{\left(1-e_{c}^{2}\right)} \cos \left(\varpi_{b}-\varpi_{c}\right)\right],
\end{aligned}
$$

where $c$ is the speed of light, and $\varpi$ is the longitude of perihelion. The above set of equations is derived from the evolution of specific angular momentum and Runge-Lenz vectors utilizing a Legendre-polynomial expansion in terms of semimajor axis ratios $\left(a_{b} / a_{c}\right)$ to octopole order (see Mardling \& Lin 2002 for the derivation), rather than the classical eccentricity expansion of the disturbing function (Laplace 1799; Brouwer $\&$ Clemence 1961). The model contains no constraints on the perturber's eccentricity, but it requires $\left(a_{b} / a_{c}\right)$ to be small. From Equations (3) to (6), it is easy to see that there exist two apsidal fixed points: $\left(\varpi_{b}-\varpi_{c}\right)=0$ and $\left(\varpi_{b}-\varpi_{c}\right)=\pi$. Each is stable for its own combination of orbital parameters, and libration around either can occur. Consequently, the system will tidally evolve toward an apsidally aligned fixed point if

$$
\frac{m_{c}}{m_{b}}>\frac{\sqrt{a_{b} / a_{c}}}{\left(1-e_{c}^{2}\right)\left(1+4\left(n_{b} a_{b} / c\right)^{2}\left(M_{\star} / m_{c}\right)\left(a_{c} / a_{b}\right)^{3}\left(1-e_{c}^{2}\right)^{3}\right.},
$$

and toward an anti-aligned state otherwise (Mardling 2007). Note that our model treats the star as a point mass, thereby neglecting its tidal and rotational deformation's contribution to the apsidal precession rates of the planetary orbits. While the rotational period of the star could not be determined from photometric observations (Butler et al. 2004), if it is short, and the star's zonal gravitational harmonics are large, such terms could provide significant corrections to the equations presented here. Nonetheless, a fast rotation rate would be extremely uncharacteristic of the star's multi-Gyr age. An additional effect that we do not consider is precession arising from the quadropole moments of the planets b and c. For ultra-short-period planets with large radii, this effect can be important, but for Gl 436b, it is small in comparison to the precession induced by the GR correction (see, e.g., Ragozzine \& Wolf 2008).

We can use our formulation to survey parameter space for regions where the perturbing planet might lie. While the age of the system is estimated to lie in the range of $\tau_{\text {sys }} \approx 1-10 \mathrm{Gyr}$ (Torres 2007), it almost certainly exceeds the few circularization timescales needed for the system to reach a fixed point. As a result, in the following analysis, we assume that the system is currently quasi-stationary, allowing us to restrict our search to fixed-point configurations.

The following scheme was used to generate fixed-point states of the system: the perturber's semimajor axis, eccentricity, and 


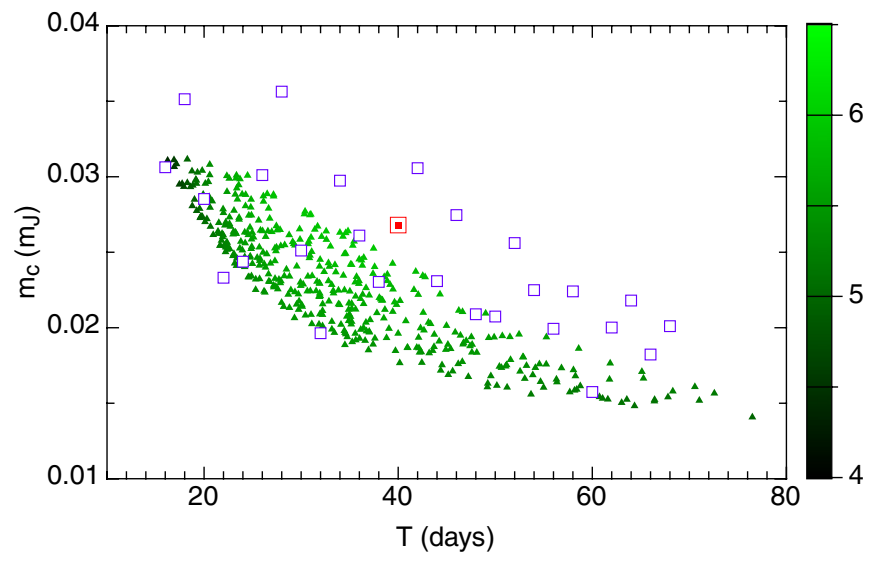

Figure 1. Distribution of masses and orbital periods for potential Gliese $436 \mathrm{c}$ candidates occupying apsidally aligned fixed points relative to the known transiting planet Gl 436b (small triangles). Color indicates the factor of increase in the orbital circularization time for Gl $436 \mathrm{~b}$ in comparison to the case where no perturber is present. The color scale runs from black (four times increase in timescale) to light green (factor of 6.5 increase in circularization time). The aligned perturbers produce significant increases in orbital circularization time, and hence are thus favored as an explanation for the substantial orbital eccentricity of Gl 436b. Aligned fixed-point models obtained using the full equations of motion are shown as open blue squares. The red dot signifies the configuration that was used for initial conditions of the numerical integration of orbital decay.

mass were chosen randomly; Equation (7) was invoked to determine whether the resulting fixed point is aligned or antialigned. By setting $d \varpi_{b} / d t=d \varpi_{c} / d t$, the eccentricity of Gl 436b was determined. If the resulting eccentricity fell between 0.149 and 0.151 , the configuration was kept. Subsequently, the generated system was tested against two constraints. First, to exclude grossly unstable systems, we required that the distance of closest approach of the two orbits was less than the radius of the larger Hill sphere, given by (Murray \& Dermott 1999)

$$
R_{H}=a(1-e)\left(\frac{m}{3 M_{\star}}\right)^{1 / 3} .
$$

In practice, this criterion will admit some unstable systems; candidate systems that make relatively close approaches can be integrated numerically to verify stability.

A planet with given values for period, mass, and eccentricity produces a radial velocity half-amplitude

$$
K=\left(\frac{2 \pi G}{P_{c}}\right)^{1 / 3}\left(\frac{M_{c} \sin (i)}{\left(M_{\star}+M_{c}\right)^{2 / 3}}\right) \frac{1}{1-e_{c}^{2}} .
$$

Consequently, we required that the radial velocity halfamplitude produced by the perturber be less than $5 \mathrm{~m} \mathrm{~s}^{-1}$ (a relatively generous criterion, even for perturbers of high eccentricity). This criterion rids the parameter space of readily detectable planets. Whenever one of our randomly generated systems satisfied our search criteria, it was numerically integrated forward in time, using the secular ODEs presented above, with the effects of tidal dissipation accounted for by including additional terms for the eccentricity and semimajor axis damping that arise from tidal dissipation in Gl 436b

$$
\begin{aligned}
\frac{d e_{b}}{d t} & =-e_{b} \frac{21 \pi}{P_{b}} \frac{k_{b}}{Q_{b}} \frac{M_{\star}}{m_{b}}\left(\frac{R_{b}}{a_{b}}\right)^{5}, \\
\frac{d a_{b}}{d t} & =-e_{b}^{2} a_{b} \frac{42 \pi}{P_{b}} \frac{k_{b}}{Q_{b}} \frac{M_{\star}}{m_{b}}\left(\frac{R_{b}}{a_{b}}\right)^{5} .
\end{aligned}
$$

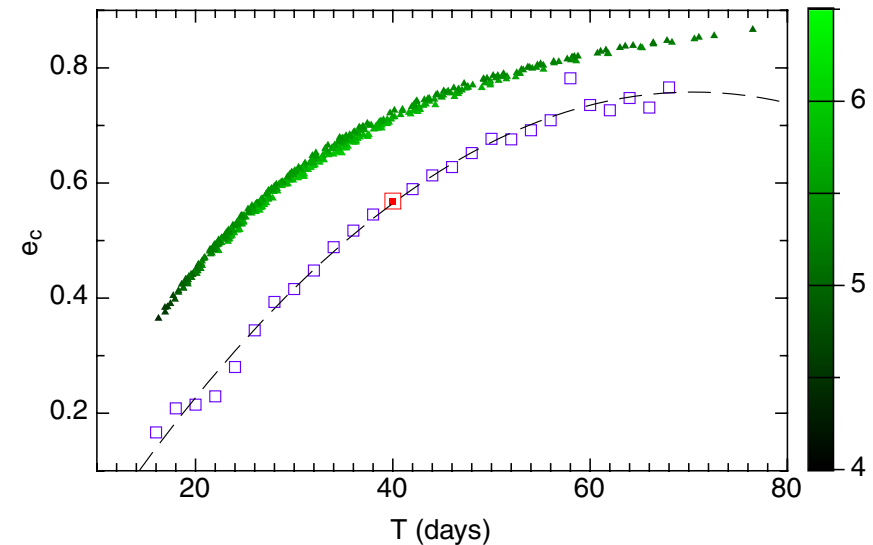

Figure 2. Distribution of eccentricities and orbital periods for potential Gliese $436 \mathrm{c}$ candidates occupying apsidally aligned fixed points relative to the known transiting planet Gl 436b (small triangles). Color indicates the factor of increase in the orbital circularization time for $\mathrm{Gl} 436 \mathrm{~b}$ in comparison to the case where no perturber is present. The color scale runs from black (four times increase in timescale) to light green (factor of 6.5 increase in circularization time). Aligned fixed-point models determined using the full equations of motion are shown as open blue squares. The red dot signifies the configuration that was used for initial conditions of the numerical integration of orbital decay. The dashed line corresponds to a best-fit second-order polynomial function (see Section 3).

For each trial system, we can thus evaluate the system's new circularization timescale by noting the time, $t$, when Gl 436b's initial eccentricity has decreased by a factor of $e$. To speed up the integration process, an artificially low $Q$ value of 300 was used. For the current purposes, we are interested less in the circularization timescale itself, but rather in the factor, $f$, by which the circularization of $b$ is increased when the perturber is added. Consequently, we divide the numerically computed timescale by that of a secular integration with only Gl $436 \mathrm{~b}$ present.

A total of 1625 model systems were generated and then integrated forward using the semianalytic model. The results of this Monte Carlo parameter survey are presented in Figures 1-4. Note that the perturbers at the anti-aligned fixed points (Figures 3 and 4) generally have smaller periods than the perturbers in aligned configurations (Figures 1 and 2). There is also a considerable difference in the factor by which the circularization timescale of planet $b$ is increased. The anti-aligned perturbers provide an average increase factor of only $1.48 \pm 0.36$. The aligned perturbers on the other hand provide an average increase factor of $5.54 \pm 0.26$. The relatively high current eccentricity of GL 436b suggests a long circularization time, thus favoring the aligned perturbers. Within the aggregate of aligned perturbers, the damping timescale increase factor varies as well, peaking around $P_{c} \approx 37 \mathrm{~d}, e_{c} \approx 0.58$, and $M_{c} \approx 8_{\oplus}$ with an increase factor of $f=6.03$. A further attractive feature of the aligned configurations is that they form a very well-defined locus in $\left(P_{c}, e_{c}, M_{c}\right)$ space. It is interesting to note that the region of parameter space occupied by viable perturbing companions is strongly influenced by the inclusion of general relativistic precession in Equation (5). With a purely Newtonian potential, apsidal alignment can only be maintained for perturbers with significantly longer orbital periods and significantly larger masses. This result is typical of many multiple-planet extrasolar systems, where one finds that the steady state dynamical configuration has a delicate dependence on the inner planet precession rate (see, e.g., Adams \& Laughlin 2006). 


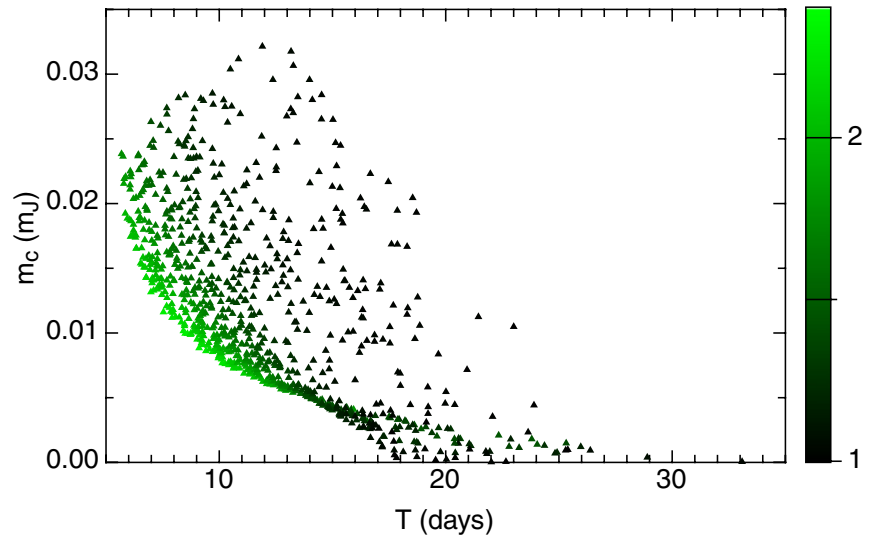

Figure 3. Distribution of masses and orbital periods for potential Gliese $436 \mathrm{c}$ candidates occupying apsidally anti-aligned fixed points relative to the known transiting planet $\mathrm{Gl} 436 \mathrm{~b}$ (small triangles). Color indicates the factor of increase in the orbital circularization time for Gl 436b in comparison to the case where no perturber is present. The color scale runs from black (no increase in timescale) to light green (factor of 3 increase in circularization time). The anti-aligned perturbers produce only modest increases in orbital circularization time, and hence are thus less favored to explain the substantial orbital eccentricity of Gl 436b.

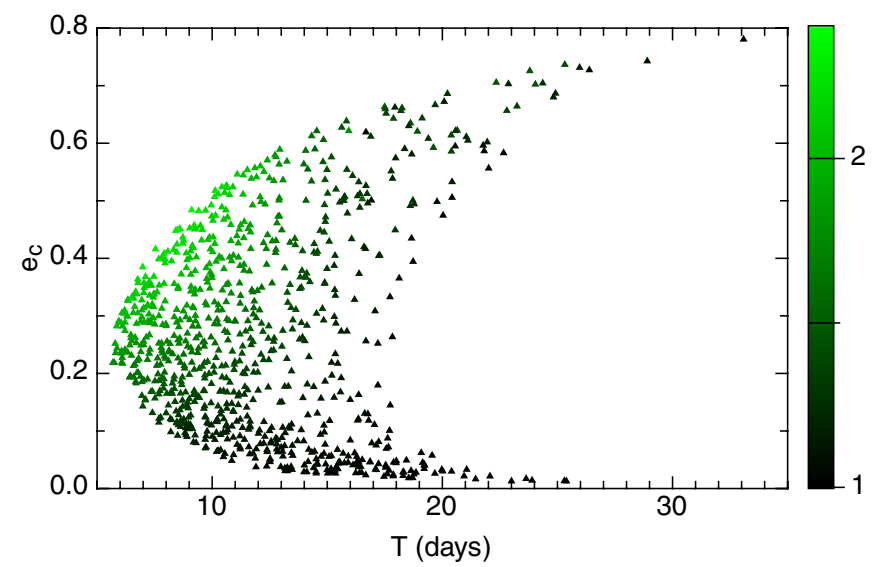

Figure 4. Distribution of eccentricities and orbital periods for potential Gliese $436 \mathrm{c}$ candidates occupying apsidally anti-aligned fixed points relative to the known transiting planet Gl 436b (small triangles). Color indicates the factor of increase in the orbital circularization time for $\mathrm{Gl} 436 \mathrm{~b}$ in comparison to the case where no perturber is present. The color scale runs from black (no increase in timescale) to light green (factor of 3 increase in circularization time).

\section{NUMERICAL CONFIRMATION}

The semianalytical approach presented above is an effective method for conducting a parameter search for potential perturbing companions and the numerical integration of the secular equations with dissipation provides an efficient procedure for evaluating the resulting increase in orbital circularization time. The octopole-order theory, however, is only approximate, and one would like to have a verification that does not incorporate the approximations. Indeed, to determine the exact fixed-point parameters of a hypothetical perturber, a fully numerical determination is much better suited. As a proof of concept, we explored 27 representative cases, each containing an apsidally aligned perturber.

The determination of the fixed-point parameters in the presence of the full equations of motion was performed by picking a perturber's period and mass, and subsequently performing many direct integrations while fine tuning the perturber's eccentricity using a Newton-Raphson scheme until a combination of orbital
Table 1

Potential Fixed-Point Perturbing Companions to Gliese 436b

\begin{tabular}{|c|c|c|c|c|}
\hline TTV (s) & $K\left(\mathrm{~m} \mathrm{~s}^{-1}\right)$ & $P$ (days) & $m\left(M_{J}\right)$ & $e$ \\
\hline 2.46 & 4.39 & 16 & $3.06298 \times 10^{-2}$ & 0.166647 \\
\hline 1.66 & 4.92 & 18 & $3.5136 \times 10^{-2}$ & 0.208449 \\
\hline 1.12 & 3.87 & 20 & $2.85267 \times 10^{-2}$ & 0.214894 \\
\hline 0.8 & 3.09 & 22 & $2.33118 \times 10^{-2}$ & 0.229319 \\
\hline 0.11 & 3.22 & 24 & $2.43739 \times 10^{-2}$ & 0.280283 \\
\hline 1.46 & 4.05 & 26 & $3.01091 \times 10^{-2}$ & 0.344026 \\
\hline 1.73 & 4.88 & 28 & $3.56371 \times 10^{-2}$ & 0.393356 \\
\hline 1.13 & 3.43 & 30 & $2.51117 \times 10^{-2}$ & 0.415769 \\
\hline 0.88 & 2.72 & 32 & $1.96387 \times 10^{-2}$ & 0.447974 \\
\hline 2.27 & 4.24 & 34 & $2.974 \times 10^{-2}$ & 0.488534 \\
\hline 0.95 & 3.79 & 36 & $2.61039 \times 10^{-2}$ & 0.517317 \\
\hline 1.37 & 3.43 & 38 & $2.30358 \times 10^{-2}$ & 0.545275 \\
\hline 2.65 & 4.07 & 40 & $2.68 \times 10^{-2}$ & 0.56866 \\
\hline 4.19 & 4.73 & 42 & $3.05687 \times 10^{-2}$ & 0.589384 \\
\hline 2.35 & 3.68 & 44 & $2.30875 \times 10^{-2}$ & 0.613239 \\
\hline 2.59 & 4.44 & 46 & $2.74619 \times 10^{-2}$ & 0.627729 \\
\hline 2.77 & 3.51 & 48 & $2.08942 \times 10^{-2}$ & 0.652055 \\
\hline 9.72 & 3.65 & 50 & $2.07423 \times 10^{-2}$ & 0.676771 \\
\hline 2.77 & 4.44 & 52 & $2.56081 \times 10^{-2}$ & 0.675824 \\
\hline 2.48 & 4.01 & 54 & $2.24914 \times 10^{-2}$ & 0.691682 \\
\hline 4.35 & 3.68 & 56 & $1.99315 \times 10^{-2}$ & 0.709095 \\
\hline 49.59 & 5.23 & 58 & $2.2408 \times 10^{-2}$ & 0.781977 \\
\hline 2.88 & 3.08 & 60 & $1.57439 \times 10^{-2}$ & 0.735554 \\
\hline 6.41 & 3.76 & 62 & $2.00128 \times 10^{-2}$ & 0.726411 \\
\hline 3.54 & 4.34 & 64 & $2.18008 \times 10^{-2}$ & 0.747703 \\
\hline 5.93 & 3.40 & 66 & $1.82263 \times 10^{-2}$ & 0.731106 \\
\hline 4.15 & 4.19 & 68 & $2.01049 \times 10^{-2}$ & 0.766115 \\
\hline
\end{tabular}

elements that corresponds to a true fixed point was reached. The Mercury6 integrator (Chambers 1999) was used. The effects of GR were modeled by adding an extra term to the star's gravitational potential that takes the form

$$
V_{\mathrm{GR}}=-3\left(\frac{G M_{\star}}{c r}\right)^{2},
$$

where $r$ is the distance from the planet to the star. The addition of the extra potential term yields the exact secular precession rate given by GR, while introducing a small error (of order $\left(\mathrm{m} / M_{\star}\right)^{2}$ ) in the planet's mean motion (Nobili \& Roxburgh 1986). The socalled hybrid algorithm was used for these integrations. All of the integrations within the Newton-Raphson iteration were short term, never exceeding $10^{4} \mathrm{yr}$.

Using this numerical scheme, we determined the fixed-point eccentricities of the potential perturbing companions, spaced with periods in equal increments between $P_{\text {lower }}=16 \mathrm{~d}$ and $P_{\text {upper }}=68 \mathrm{~d}$. In Table 1 , we list the resulting systems (which are also plotted as a sequence of blue squares on Figures 1 and 2). The perturber's eccentricities can be approximated by a second-order polynomial function: $e_{c} \approx-0.277+2.94 \times$ $10^{-2} P_{c}-2.05 \times 10^{-4} P_{c}^{2}$, where the period is measured in days (plotted as a dashed line on Figure 2). It is clear that the inclusion of the full disturbing function produces some corrections to the octopole-order analytic solutions. Namely, secular theory tends to overestimate the fixed-point eccentricities of the perturbers for this system.

Table 1 lists TTVs, $\Delta T_{\max }$ for the fixed-point systems. The amplitude of the timing variations changes by a few seconds along this locus of models in $\left(P_{c}, e_{c}, M_{c}\right)$ space. Much of this variation is due to the given configuration, residing close to, or in a high-order MMR. An extreme example is a $P_{c}=58 \mathrm{~d}$ perturber system, where the planets reside in a 22:1 resonance. Due 


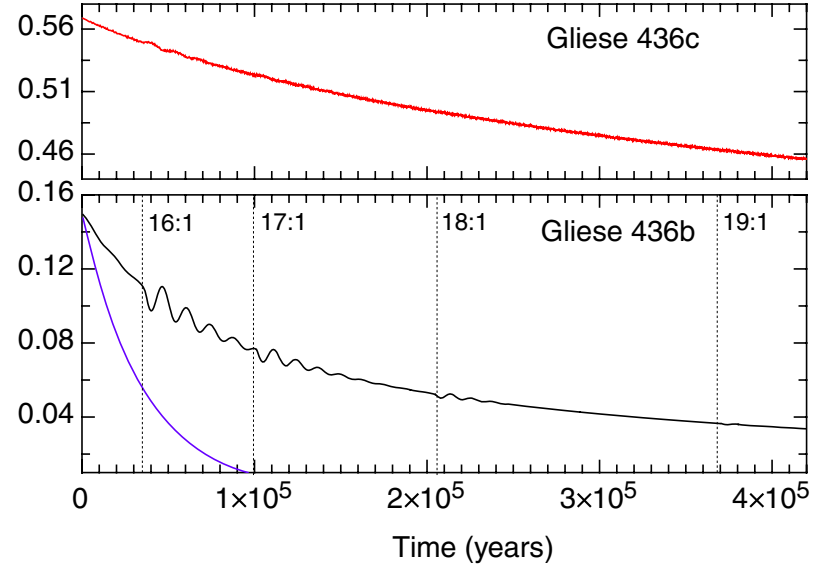

Figure 5. Numerically computed eccentricity evolution of the tidally dissipated Gliese 436 system, starting from a numerically determined aligned fixed point. The top plot corresponds to the eccentricity of a $P=40 \mathrm{~d}$ perturber. The black curve in the bottom plot corresponds to the eccentricity evolution of Gl 436b, in presence of the perturber. The blue curve in the bottom plot corresponds to the scenario where Gl 436b is alone. For the integrations, $Q=10$ was used.

to the perturber's very high fixed-point eccentricity, TTVs reach almost a minute. Hence, if our theory is correct, a given measurement of $\Delta t_{\text {max }}$ may provide an immediate hint to $P_{c}, e_{c}$, and $M_{c}$.

Our iteratively determined fixed-point models all appear to be indefinitely dynamically stable. By definition, at the fixed point, orbital parameter variations are reduced to the slow tidal circularization of both orbits, and so the planets never get into trouble. Interestingly, however, for eccentricities $e_{c} \gtrsim 0.8$, we find that the octopole-order fixed-point solutions, when integrated using the full equations of motion, are largely unstable. When given access to the full disturbing function, these configurations usually experience eccentricity variations and librations in $\varpi_{c}-\varpi_{b}$ large enough to generate orbit crossings. The true fixed-point configurations thus represent islands of stability within a larger sea, yielding a potentially important clue to the initial formation and evolution of the Gliese 436 planetary system. Indeed, if our model is confirmed by observation, then the question of system formation will represent an interesting line of inquiry.

Finally, we carried out a full numerical demonstration of the circularization timescale of the two-planet $P_{c}=40 \mathrm{~d}$ system listed on the thirteenth line of Table 1. This system lies near the region where the secular ODEs exhibit the largest factors of increase, $f$. To carry out the full integrations, we used a selfconsistent numerical scheme that in addition to gravitational dynamics, takes into account the effects of tidal damping and spin evolution (Eggleton et. al 1998; Mardling \& Lin 2002). In this simulation, the post-Newtonian gravitational force was computed in the same way as above. The tidal force takes the form

$$
\begin{aligned}
\mathbf{F}_{T}= & -\left(\frac{6 n\left(k_{2} / 2\right)}{Q}\right)\left(\frac{M_{\text {star }}}{m}\right)\left(\frac{R}{a}\right)^{5}\left(\frac{a}{|r|}\right)^{8} \\
& \times\left[3(\hat{\boldsymbol{r}} \cdot \dot{\boldsymbol{r}}) \hat{\boldsymbol{r}}+\left(\hat{\boldsymbol{r}} \times \dot{\boldsymbol{r}}-r \boldsymbol{\Omega}_{b}\right) \times \hat{\boldsymbol{r}}\right]
\end{aligned}
$$

and the spin evolution is governed by

$$
\dot{\boldsymbol{\Omega}}=\frac{m M_{\star}}{m+M_{\star}}\left(\frac{\boldsymbol{r} \times \mathbf{F}_{T}}{I}\right),
$$

where $\Omega$ is the spin vector and $I$ is the planet's moment of inertia. Note that, as before, tidal dissipation is applied only to Gl $436 \mathrm{~b}$. As a result, Gl 436b's $Q$ value absorbs all other dissipation in the

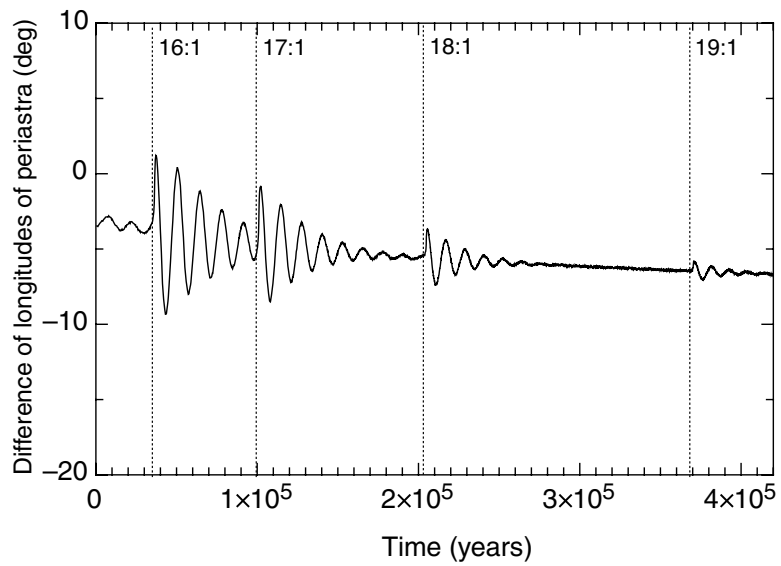

Figure 6. Difference between apsidal lines of Gl 436b and c for the numerically computed tidally dissipated eccentricity evolution shown in Figure 5.

system. Nonetheless, it is likely that the contribution from the perturber will be comparably small, since $d E / d t \propto(R / a)^{5}$, as will be the tides raised on the star where $d E / d t \propto\left(m / M_{\star}\right)$. Here also, an artificially low $Q$ value of 10 (characteristic of Earth-like planets) was used in order to speed up the integration process. Integration was done using the Bulirsch-Stoer algorithm (Press et al. 1992) with a time step accuracy parameter $\eta=10^{-14}$. The moment of inertia was taken as $I / M_{\mathrm{b}} R_{\mathrm{b}}^{2}=0.254$ and the initial spin frequency was $\Omega=2.3 \mathrm{~d}$. As we have done throughout the paper, we use $P_{b}=2.643 \mathrm{~d}, e_{b}=0.15$, and $m_{b}=0.0711 M_{J}$.

Two scenarios were computed: a tidally dissipated Gliese 436 system with and without a fixed-point $P_{c}=40 \mathrm{~d}$ perturber. Figure 5 shows the eccentricity evolutions of the perturbed and unperturbed Gl 436b's as well as the eccentricity evolution of the perturber. The results of this integration are in good agreement with the semianalytical integrations discussed in Section 2. By measuring the time at which Gl 436b's eccentricity dropped by a factor of $e$ in the perturbed and unperturbed solutions, we find that the $P_{c}=40 \mathrm{~d}$ perturber provides a $f \sim 5.3$ times increase in the circularization timescale. Figure 6 shows the difference of perihelia, $\left(\varpi_{b}-\varpi_{c}\right)$ as a function of time. Linear secular theory predicts that once the fixed point is reached, the system does not deviate away from it at all. In the full numerical integration, we observe some low-amplitude libration, which is associated with passage of Gl 436b through high-order MMRs (namely, 16:1, 17:1, 18:1, and 19:1 as labeled on Figures 5 and 6).

\section{MANY-PLANET FIXED POINTS}

Up to this point, we have only considered a scenario where the Gl 436b's eccentricity is maintained by a single perturber. While a two-planet scenario is the simplest one, it is certainly possible to obtain solutions where three or more planets are all in a quasi-stationary configuration. In this case, to fully take advantage of the energy reservoir of the system, all consecutive pairs of planets should be dynamically coupled. Let us now examine fixed-point systems in the context of a modified classical Laplace-Lagrange secular theory.

To second order in eccentricities and first order in masses, the averaged co-planar disturbing function of planet $i$ can be written as

$$
\left\langle\mathcal{R}_{i}^{(\mathrm{sec})}\right\rangle=n_{i} a_{i}^{2}\left[\frac{1}{2} A_{i i} e_{i}^{2}+\sum_{j=1, j \neq i}^{N} A_{i j} e_{i} e_{j} \cos \left(\varpi_{i}-\varpi_{j}\right)\right] .
$$


Table 2

An Example Three-Planet Fixed-Point Configuration

\begin{tabular}{lcccc}
\hline \hline Planet & $P($ days $)$ & $m\left(M_{J}\right)$ & $e$ & $\varpi(\mathrm{deg})$ \\
\hline $\mathrm{b}$ & 2.64 & $7.11 \times 10^{-2}$ & 0.15 & 0.0 \\
$\mathrm{c}$ & 14.24 & $6.92 \times 10^{-2}$ & 0.139214 & 0.0 \\
$\mathrm{~d}$ & 27.33 & $6.92 \times 10^{-2}$ & 0.152183 & 0.0 \\
\hline
\end{tabular}

The constant coefficients $A_{i j}$ form a $N \times N$ matrix, which renders the dynamics of the system (Murray \& Dermott 1999). Here, the effects of GR are accounted for by introducing a leading-order correction to the diagonal elements of the $\boldsymbol{A}$ matrix (for details on this standard modification, see Adams \& Laughlin 2006). As a result, the elements take the form

$$
\begin{gathered}
A_{i i}=\frac{n_{i}}{4} \sum_{j=1, j \neq i}^{N} \frac{m_{j}}{M_{\star}+m_{i}} \alpha_{i j} \bar{\alpha}_{i j} b_{3 / 2}^{(1)}\left(\alpha_{i j}\right)+3 n_{i} \frac{G M_{\star}}{c^{2} a_{i}}, \\
A_{i j}=-\frac{n_{i}}{4} \sum_{j=1, j \neq i}^{N} \frac{m_{j}}{M_{\star}+m_{i}} \alpha_{i j} \bar{\alpha}_{i j} b_{3 / 2}^{(2)}\left(\alpha_{i j}\right),
\end{gathered}
$$

where $\alpha_{i j}=a_{i} / a_{j}$ if $\left(a_{i}<a_{j}\right) ; a_{j} / a_{i}$ if $\left(a_{j}<a_{i}\right), \bar{\alpha}_{i j}=\alpha_{i j}$ if $\left(a_{i}<a_{j}\right) ; 1$ if $\left(a_{j}<a_{i}\right), b_{3 / 2}^{(1)}\left(\alpha_{i j}\right) \& b_{3 / 2}^{(2)}\left(\alpha_{i j}\right)$ are Laplace coefficients of first and second kind, respectively. The lowestorder Lagrange's equations of motion can be written as

$$
\begin{aligned}
\frac{d e_{i}}{d t} & =-\frac{1}{n_{i} a_{i}^{2} e_{i}} \frac{\partial \mathcal{R}_{i}}{\partial \varpi_{i}}=\sum_{j=1, j \neq i}^{3} A_{i j} e_{j} \sin \left(\varpi_{i}-\varpi_{j}\right), \\
\frac{d \varpi_{i}}{d t} & =\frac{1}{n_{i} a_{i}^{2} e_{i}} \frac{\partial \mathcal{R}_{i}}{\partial e_{i}}=A_{i i}+\sum_{j=1, j \neq i}^{3} A_{i j} \frac{e_{j}}{e_{i}} \cos \left(\varpi_{i}-\varpi_{j}\right) .
\end{aligned}
$$

From these expression, it can be seen that $d e / d t$ vanishes when the orbits are apsidally aligned and anti-aligned. Since all precession rates of a fixed-point system are equal, given the masses and semimajor axes of the perturbing planets, we can solve for their fixed-point eccentricities. Care must be taken in this approach however, since Laplace-Lagrange theory only applies in the limit of small eccentricities. ${ }^{5}$

Let us now consider a single proof-of-concept calculation. Setting the masses of the two perturbers at $22 m_{\oplus}$ each, we allowed the semimajor axes to be generated randomly, and then solved for the perturbers' aligned fixed-point eccentricities. We then used the numerical scheme discussed above to integrate the full equations of motion forward in time. The orbital elements of this configuration are presented in Table 2, whereas Figure 7 shows the system's evolution toward a triply circular state. Since the eccentricities of this configuration are somewhat higher than what is required for Laplace-Lagrange theory to reach high levels of precision, it is not surprising that the analytically obtained fixed point is only approximately stationary, and some eccentricity modulation in the numerical integration is observed.

In principle, a similar survey to that described in Section 2 can be performed. However, as more planets are added, the parameter space, available for exploration, becomes overwhelmingly large. Thus, such a search would be most effective in case a perturbing planet is already found, but is clearly not at a singleperturber fixed point. Indeed, such a detection may indirectly hint to the presence of additional, dynamically significant, bodies in the system.

\footnotetext{
5 The issue of small eccentricities can be forestalled by expanding the secular disturbing function to higher order in $e$.
}

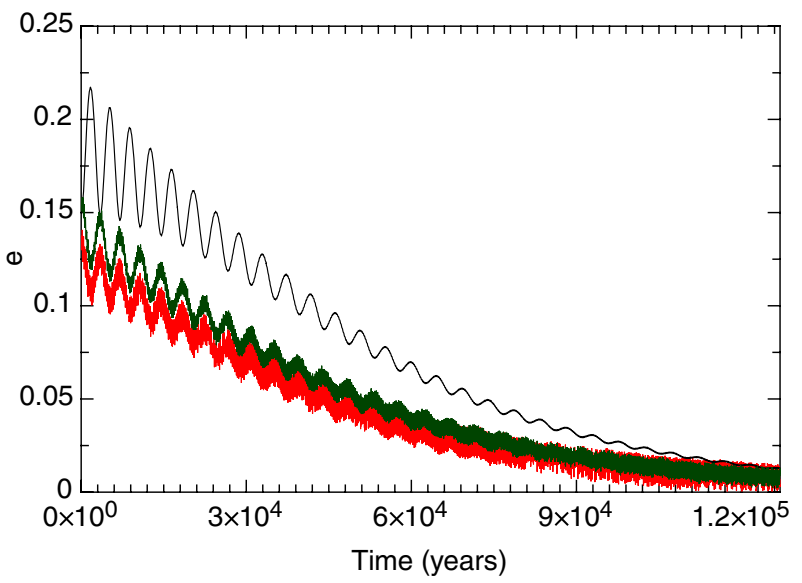

Figure 7. Numerically computed eccentricity evolution of the tidally dissipated Gliese 436 system with three planets. The starting conditions were computed analytically, and are listed in Table 2 . The black, red, and green curves correspond to planets $\mathrm{b}$, c, and d, respectively. For the integrations, $Q=8$ was used.

\section{DISCUSSION}

In this paper, we put forward the hypothesis that the Gliese 436 system has an additional, as-yet undetected companion, Gliese 436c, that is not in a low-order mean motion resonance with Gl 436b, but is at a secular fixed point. An important role of such a perturber would be to increase the tidal circularization timescale of the system and thus help explain the unusually high eccentricity, $e=0.15$, of Gl $436 \mathrm{~b}$.

Using the dissipated secular theory developed by Mardling (2007), we surveyed the parameter space of allowed perturbers, restricting our domain to fixed-point configurations. This survey revealed that apsidally aligned, fixed-point systems can provide significantly longer circularization times than the anti-aligned states, and are thus preferred. Furthermore, the population of possible stationary, apsidally aligned, perturbers forms a clear and well-defined locus in $\left(P_{c}, M_{c}, e_{c}\right)$ space. This sequence is accompanied by the amplitude of TTVs, $\Delta T_{\max }$, listed in Table 1 . As a check of the validity of our semianalytic theory, we verified that the full disturbing function produces only small corrections to the orbital elements of the fixed-point configurations derived from octopole theory. In addition, we performed one self-consistent numerical integration of the circularization, a hypothetical $P_{c}=40 \mathrm{~d}$ perturber. The results are in good agreement with the octopole theory, allowing us to verify that a factor, $f=5.3$ increase in circularization timescale is robust.

A satisfactory theory makes predictions that are not only testable, but readily testable. The presence of Glise 436c, with the orbital and physical properties that we are proposing would fall into the readily testable category.

We make several predictions. (1) If a secular perturber exists and holds significant responsibility for the upkeep of Gl 436b's eccentricity, we believe that it will fall on the loci shown in Figures 1 and 2 and delineated in Table 1. (2) Our theory gives a roughly sixfold increase in the circularization timescale derived by Tong $\&$ Zhou (2009). If we adopt $Q=300,000$, consistent with the upper bound on Banfield \& Murray's (1992) estimate of Neptune's $Q$ value, and near the lower bound on Jupiter's $Q$ value, then the effective (fixed-point) circularization timescale becomes $\tau \sim 8 \mathrm{Gyr}$, a value that modestly exceeds the likely age of the star. In addition, the system may also require up to $\sim 3$ single-planet circularization timescales to arrive at the fixed point. As a result, for the first time, we do not need to invoke an 
uncomfortably high $Q$ value to explain the observed eccentricity, but if our theory is correct then Gl 436b's effective internal dissipation is a factor of 10 times less than Uranus and is near the lower end of Neptune's range, suggesting a fundamental structural difference between the "hot Neptune" and the slightly less massive solar system "ice giants." (3) We predict that Gl $436 \mathrm{~b}$ will show no measurable tidal heating. On the surface, this appears inconsistent with the Deming et al. (2007) measurement of $T=712 \pm 36 \mathrm{~K}$ for the planet's $8 \mu \mathrm{m}$ brightness temperature. If our theory is correct, the apparent temperature excess over the $T_{\mathrm{eq}}=642 \mathrm{~K}$ planetary effective temperature will arise from a non-blackbody emission spectrum, weather variability, measurement uncertainty, or a combination of the three.

In the previous section, we reviewed the possibility of more than one perturber maintaining Gl 436b's eccentricity, utilizing modified Laplace-Lagrange secular theory, and performed one numerical integration as an example. In light of this possibility, we note that observational detection of a companion not in a twoplanet fixed configuration may indirectly point at the presence of additional planets in the system.

What are the prospects for near-term observational detection of Gliese $436 \mathrm{c}$ ? During the two yr following the radial velocity analysis of Maness et al. (2007), the Gliese 436 system has been placed under intensive radial velocity surveillance. At the IAU 253 symposium in 2008 May, the Geneva Planet Search team (Lovis et al. 2008) showed several dozen new high-precision Doppler measurements, which indicated that the possible longterm linear trend noted by Maness et al. (2007) has either abated, or was an artifact of the limited observational baseline. A residual periodogram of the Geneva team's velocity data showed a number of peaks in the $10 \mathrm{~d}<P<100 \mathrm{~d}$ range, several of which appeared tall enough to correspond to a perturber with the properties required by our theory.

The EPOXI mission (Deming et al. 2008) held Gliese 436 under continuous photometric observation for $27 \mathrm{~d}$ during 2008 May. The full results of their investigation have not yet been published, but the EPOXI transit timing precision for Gl $436 \mathrm{~b}$ is expected to be of the order of a few tens of seconds (Deming 2009). At this level of precision, if our hypothesis of a fixed-point perturber is correct, we predict that no TTVs will be observed by EPOXI. Thus far, our hypothesis has been consistent with the lack of transit timing variations above $\Delta t \sim 45 \mathrm{~s}$ reported by Coughlin et al. (2008). If TTVs do exist, their maximum amplitude will lie in the neighborhood of $\Delta t \sim 1$ to $\Delta t \sim 10 \mathrm{~s}$, and their maximum amplitude will give a strong indication of the perturber's orbital properties, as interpolated from Table 1.

Recent improvements in high-cadence, high-precision ground-based photometry have improved the measurement of transit midpoints to within $\Delta t \sim 5 \mathrm{~s}$ (see, e.g., Johnson et al. 2008). If this level of precision can be repeatedly applied to Gliese 436, then there is a significant chance that the presence of a perturbing planet can be inferred. In addition, the predicted radial velocity half-amplitudes, $K$, of the perturber are generally well within the current threshold of detection, which should enable a ready confirmation or falsification of our hypothesis.

We thank Fred Adams, Dan Fabrycky, Rosemary Mardling, Stan Peale, David Stevenson, Darin Ragozzine, and Drake Deming for useful discussions.

This research is based in part upon work supported by the National Science Foundation CAREER program under grant
0449986 (G.L.), NSF AST-0307493 Grant (S.V.), and by NASA through the Ames Astrobiology Institute under cooperative agreement NNA06CB31A.

\section{REFERENCES}

Adams, F. C., \& Laughlin, G. 2006, ApJ, 649, 1004

Agol, E., Steffen, J., Sari, R., \& Clarkson, W. 2005, MNRAS, 359, 567

Alonso, R., Barbieri, M., Rabus, M., Deeg, H. J., Belmonte, J. A., \& Almenara, J. M. 2008, A\&A, 487, 5

Banfield, D., \& Murray, N. 1992, Icarus, 99, 390

Bean, J. L., et al. 2008, A\&A, 486, 1039

Brouwer, D., \& Clemence, G. M. 1961, Methods of Celestial Mechanics (New York: Academic Press)

Butler, R. P., Vogt, S. S., Marcy, G. W., Fischer, D. A., Wright, J. T., Henry, G. W., Laughlin, G., \& Lissauer, J. J. 2004, ApJ, 617, 580

Chambers, J. E. 1999, MNRAS, 304, 793

Coughlin, J., Stringfellow, G., Becker, A., Lopez-Morales, M., Mezzalira, F., \& Krajci, T. 2008, ApJ, 689, L149

Deming, D., et al. 2008, BAAS, 40, 202

Deming, D. 2009, in IAU Symp. 253, Transiting Planets, ed. Frédéric Pont, D. Sasselov, \& M. Holman (Cambridge: Cambridge Univ. Press), 197

Deming, D., Harrington, J., Laughlin, G., Seager, S., Navarro, S. B., Bowman, W. C., \& Horning, K. 2007, ApJ, 667, L199

Demory, B.-O., et al. 2007, A\&A, 475, 1125

Eggleton, P. P., Kiseleva, L. G., \& Hut, P. W. 1998, ApJ, 499, 853

Fortney, J. J., Marley, M. S., \& Barnes, J. W. 2007, ApJ, 659, 1661

Gillon, M., et al. 2007, A\&A, 472, L13

Goldreich, P., \& Peale, S. 1966, AJ, 71, 425

Goldreich, P., \& Soter, S. 1966, Icarus, 5, 375

Holman, M. J., \& Murray, N. W. 2005, Science, 307, 1288

Hut, P. 1981, A\&A, 99, 126

Jackson, B., Barnes, R., \& Greenberg, R. 2008a, in AAS/Division for Planetary Sciences Meeting Abstracts 40, \#04.02, Bulletin of the American Astronomical Society, 40, 391

Jackson, B., Greenberg, R., \& Barnes, R. 2008b, ApJ, 678, 1396

Johnson, J. A., Winn, J. N., Cabrera, N. E., \& Carter, J. A. 2008, arXiv:0812.0029

Laplace, P. S. 1799, Traitede Mecanique Celeste (Paris: Gauthier-Villars)

Levrard, B., Correia, A. C. M., Chabrier, G., Baraffe, I., Selsis, F., \& Laskar, J. 2007, A\&A, 462, L5

Lovis, C., Mayor, M., Bouchy, F., Pepe, F., Queloz, D., Udry, S., Benz, W., \& Mordasini, C. 2008, Proc. IAU Symp. S253, Vol. 4 (Dordrecht: Kluwer), 502

Maness, H. L., Marcy, G. W., Ford, E. B., Hauschildt, P. H., Shreve, A. T., Basri, G. B., Butler, R. P., \& Vogt, S. S. 2007, PASP, 119, 90

Mardling, R. A. 2007, MNRAS, 382, 1768

Mardling, R. A. 2008, MNRAS, submitted (arXiv:0805.1928)

Mardling, R. A., \& Lin, D. N. C. 2002, ApJ, 573, 829

Matsumura, S., Takeda, G., \& Rasio, F. A. 2008, ApJ, 686, 1

Mayor, M., \& Queloz, D. 1995, Nature, 378, 355

Meyer, J., \& Wisdom, J. 2008, Icarus, 193, 213

Murray, C. D., \& Dermott, S. F. 1999, Solar System Dynamics (Cambridge: Cambridge Univ. Press) (MD99)

Nobili, A., \& Roxburgh, I. W. 1986, in IAU Symp. 114, Relativity in Celestial Mechanics and Astrometry, ed. J. Kovalevsky \& V. A. Brumberg (Dordrecht: Reidel), 105

Peale, S. J., \& Cassen, P. 1978, Icarus, 36, 245

Peale, S. J., \& Greenberg, R. J. 1980, in Lunar and Planetary Institute Conf. Abstr., 11, 871

Pont, F., Gilliland, R. L., Knutson, H., Holman, M., \& Charbonneau, D. 2009, MNRAS, 393, L6

Press, W. H., Teukolsky, S. A., Vetterling, W. T., \& Flannery, B. P. 1992, Numerical Recipes in FORTRAN: The Art of Scientific Computing (Cambridge: Cambridge Univ. Press)

Ragozzine, D., \& Wolf, A. S. 2008, in press (arXiv:0807.2856)

Ribas, I., Font-Ribera, A., \& Beaulieu, J.-P. 2008, ApJ, 677, L59

Tittemore, W. C., \& Wisdom, J. 1989, Icarus, 78, 63

Tong, X., \& Zhou, J. 2009, Science in China (G): Physics, Mechanics \& Astronomy, 52, 640

Torres, G. 2007, ApJ, 671, L65

Winn, J. N., et al. 2005, ApJ, 631, 1215

Wisdom, J. 2008, Icarus, 193, 637

Wu, Y. 2003, in Scientific Frontiers in Research on Extrasolar Planets, ed. D. Deming \& S. Seager (San Francisco, CA: ASP), in press

Zhang, K., \& Hamilton, D. P. 2008, Icarus, 193, 267 